\title{
Well Matched Electromagnetic Boundary in FDTD-PIC for Charged Particle Penetration
}

\author{
Lars D. Ludeking* and Andrew J. Woods*
}

ATK-Mission Systems Group, Newington, VA 22122, USA

\begin{abstract}
The authors address the Matched Phase Velocity method for bounding an electromagnetic (EM) domain in the MAGIC finite difference-time domain (FDTD) particle-in-cell (PIC) EM code. Reflected power is shown to be reduced 18 $\mathrm{dB}$ compared to the conventional port treatment. Boundary quality is preserved under penetration by charged particles.
\end{abstract}

Keywords: MAGIC, EM FDTD PIC code, matched phase velocity, advection, EM boundary.

\section{INTRODUCTION}

Simulations with Finite-Difference-Time-Domain Particlein-Cell (FDTD-PIC) computational methods provide insight into a broad spectrum of electromagnetic field and plasma beam wave applications. One of the principal challenges is the correct modeling of the EM boundary at terminations of the simulation space. There is a rich field of methods used in the termination of an electromagnetic domain [1]. These methods may collectively be regarded in general as "absorbing wave boundary conditions", or ABC's. As a group, however, many of the more sophisticated methods impose an artificial loss term to provide damping of the exiting wave field. These methods often provide excellent broadband absorption of outgoing wave fields, but generally form two liabilities with respect to PIC simulations. First, the methods are by design purely absorbing and provide no simple approach for injection of wave fields into a simulation. And secondly, they are typically unstable or at best degraded by the impingement of the PIC-macroparticles through the boundary zone. Historically, a very simple Matched Phase Velocity (MPV) has been found suitable for both conditions [2,3]. We will begin with a simple improvement to the Matched Phase Velocity method that is based on the 1-dimensional advection equation approach $[4,5]$. Simply stated, this method ensures proper centering of the boundary equation in time and space in the direction of wave propagation, as such we will refer to this as the Centered Matched Phase Velocity (CMPV) method. (In future work, we intend to look at the additional multidimensional effects suggested in the approach of EnquistMajda [5] to the uni-directional wave equation.)

In the following discussion we will be using the FDTDPIC code MAGIC to illustrate the effectiveness of the approach that we are developing. The MAGIC software is a user-configurable EM FDTD-PIC simulation code used for the modeling and simulation of beam wave interactions and electro-energetic processes between space charge and EM

*Address correspondence to these authors at the ATK-Mission Systems Group, Newington, VA 22122, USA; Tel: (703)-254-2416; Fax: (703)-3396953; E-mails: lars.ludeking@atk.com, andrew.woods@atk.com fields. This approach is versatile and provides self consistent interaction between particles and fields. The MAGIC software includes a broad variety of boundary and material properties as well as particle and field algorithms. We will make use of only a selected subset to illustrate the behavior of the improved CMPV approach.

The centered MPV method for bounding an electromagnetic domain will be shown to be effective in reducing unwanted boundary reflections in the MAGIC EM code and has the pleasing symmetry of application that waves may be injected and removed from the simulation via the same numerical methodology, and does not exhibit the unstable behavior of other $\mathrm{ABC}$ methods when penetrated by charged particles.

\section{MAXWELL SOLVER TERMINATION}

In FDTD-PIC perfect conductor and periodic and mirror symmetries are easily treated both with and without particles. However, the open (or unconstrained) boundary termination is the case which has received the most attention and required more care when particles are allowed to exit the simulation, or conversely are injected into the simulation space through the "open boundary".

There are two crucial issues: first the completion of the simulation domain with an effective one-sided wave equation, or uni-directional wave equation (i.e. it closes or completes Maxwell's equations on the simulation edge); secondly, is the boundary truncation insensitive (transparent) to the passage of ambient particles through the boundary?

For the first issue, the closure of Maxwell's equations, there are several competing approaches, each with its own advantages and disadvantages. Four of the methods implemented in MAGIC are:

- $\quad$ The matched phase velocity method [2, 3], (MPV),

- $\quad$ The centered matched phase velocity method $[4,5]$, (CMPV),

- $\quad$ The optimized free space method [6], and

- $\quad$ The CPML method [7].

For this paper we will consider only a variant of the matched phase velocity method, since the other two 
approaches are generally impractical when particles penetrate the absorption region.

The matched phase velocity method (MPV), while historically the oldest approach, is in general the most robust for two important aspects of simulation modeling, beam wave propagation and the boundary termination with wave injection. The simplified form of the MPV in 1-dimension is listed below for the outgoing wave formulation.

$E_{0}^{n+1}=(1-\beta) E_{0}^{n}+\beta E_{1}^{n+1}$

$\beta=\frac{\frac{c \Delta t}{\Delta x}}{\sqrt{\varepsilon_{r} \mu_{r}}}$

The centered matched phase velocity (CMPV) method separates artificial numerical dispersion from physical dispersion and compensates for the computational artifact. It begins by writing the hyperbolic one dimensional wave propagation equation in (Cartesian) operator form as follows:

$\left(\frac{\partial^{2}}{\partial x^{2}}-\mu \varepsilon \frac{\partial}{\partial t^{2}}\right) E=0$

This may be factored as follows.

$$
\left(\frac{\partial}{\partial x}+\sqrt{\mu \varepsilon} \frac{\partial}{\partial t}\right)\left(\frac{\partial}{\partial x}-\sqrt{\mu \varepsilon} \frac{\partial}{\partial t}\right) E=\mathbf{0} \text {. }
$$

Note that the wave equation is separated into a forward and backward wave operator and that for a wave propagating in one direction only the forward or backward operator results in zero. Consider only the portion of the operator that is the solution to a forward traveling $(+\mathrm{x})$ wave, then we can write the following operation equation

$\left(\frac{\partial}{\partial x}+\sqrt{\mu \varepsilon} \frac{\partial}{\partial t}\right)^{N} E=0$

For $\mathrm{N}=1$, the standard wave equation results (also identified as the advection equation.) However, it is permissible to operate on the wave field multiple times and still satisfy the wave equation. Thus in the finite difference representation, it is implicit that for a reasonable approximate value of the phase velocity, the first order equation is approximately zero, and one expects that multiple applications of the difference operator will provide improved results, leading to a better approximation to a perfect match.

For boundary termination, two complementary aspects of this method are of value in MAGIC; these are the separation of the scattered wave component and the incident wave component. Fig. (1) illustrates the finite difference representation of the field components that contribute to the update at the latest time step and at the edge of the simulation domain for the operator that gives a solution to a negatively traveling wave.

Using a temporal and spatially centered forms for the finite difference approximation to the differential equation we can write the finite difference form as

$E_{0}^{n+1}=E_{1}^{n}+f\left(E_{1}^{n+1}-E_{0}^{n}\right)$. $\beta=\frac{\frac{c \Delta t}{\Delta x}}{\sqrt{\varepsilon_{r} \mu_{r}}}$.

$f=\frac{\beta-1}{\beta+1}$.

the first order $(\mathrm{n}=1)$ solution to the operator equation, requires knowledge of the boundary value at the preceding time step and the values of the field one spatial step to the interior both for the current and the preceding time step. Application of higher order solutions requires information further into the simulation and farther back in time. Thus for $\mathrm{n}=1$, we need three time-space fields to obtain the edge value. For $n=2$, we require eight time-space fields, and so forth to higher order.

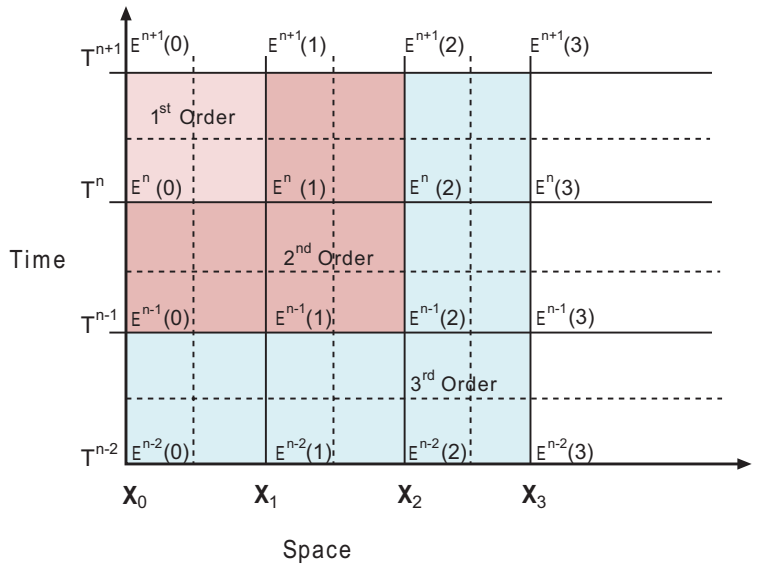

Fig. (1). This illustrates the contributing fields for the $1^{\text {st }}, 2^{\text {nd }}$ and $3^{\text {rd }}$ order CMPV method.

Before proceeding to the higher order results, we will introduce finite difference operators and recast the differential form accordingly.

$E_{m}^{n+1}=I E_{m}^{n+1}$, this is the identity operator.

$E_{m+1}^{n+1}=X E_{m}^{n+1}$, this is the spatial promotion operator.

$E_{m}^{n}=T E_{m}^{n+1}$, this is the temporal demotion operator.

The finite difference advection operation may be written as the following

$(I-X T+f T-f X) E_{0}^{n+1}=\mathbf{0}$.

Applying the advection operation a second time gives

$(I-X T+f T-f X)(I-X T+f T-f X) E_{0}^{n+1}=\mathbf{0}$.

this yields the following $2^{\text {nd }}$ order finite difference update for the boundary value.

$$
\begin{aligned}
E_{0}^{n+1}= & -E_{2}^{n-1}+2 f\left(E_{1}^{n+1}+E_{1}^{n-1}-E_{0}^{n}-E_{2}^{n}\right) \\
& -f^{2}\left(E_{2}^{n+1}+E_{0}^{n-1}\right)+2\left(1+f^{2}\right) E_{1}^{n} .
\end{aligned}
$$
times.

Continuing this process we apply the operator three

$$
\begin{gathered}
(I-X T+f T-f X)(I-X T+f T-f X) \\
(I-X T+f T-f X) E_{0}^{n+1}=0
\end{gathered}
$$




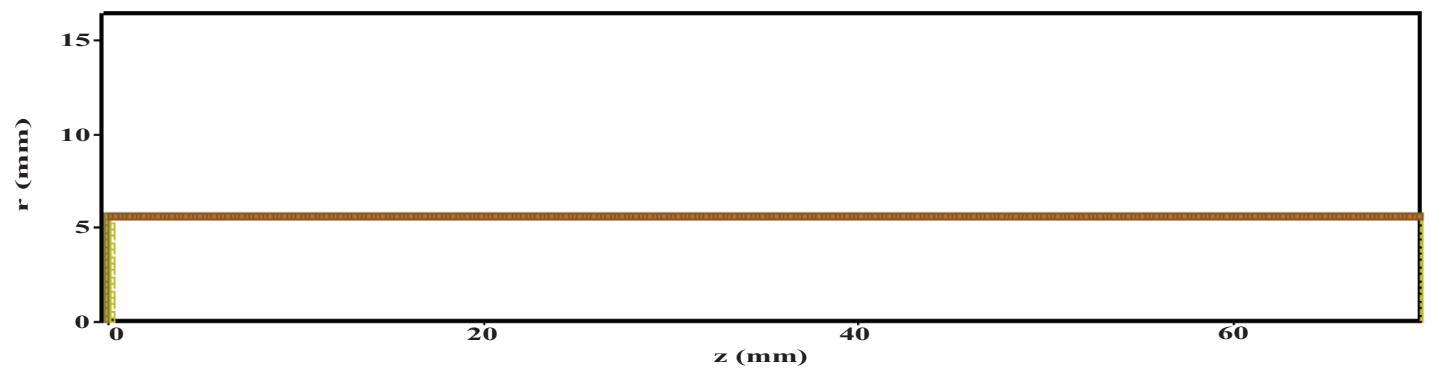

Fig. (2). Plane (rz) of a circular waveguide with $103 \mathrm{GHz}$ TE01 excitation at the right end.

After considerable rework we obtain the $3^{\text {rd }}$ order finite difference update equation for the boundary value. (Note that this requires 15 time and space values to obtain the $16^{\text {th }}$ value lying on the boundary. There are 4 spatial locations, corresponding to 3 full spatial cells. And there are 4 temporal locations, 1 current time at all spatial locations and values at 3 earlier time steps.)

$$
\begin{aligned}
E_{0}^{n+1}= & E_{3}^{n-2}+3 f\left(E_{1}^{n+1}-E_{0}^{n}+E_{3}^{n-1}-E_{2}^{n-2}\right)+ \\
& 3 f^{2}\left(E_{2}^{n+1}-E_{3}^{n}+E_{0}^{n-1}-E_{1}^{n-2}\right)+ \\
& 3\left(1+2 f^{2}\right)\left(E_{1}^{n}-E_{2}^{n-1}\right)+3 f\left(2+f^{2}\right)\left(E_{2}^{n}-E_{1}^{n-1}\right)+ \\
& f^{3}\left(E_{3}^{n+1}-E_{0}^{n-2}\right)
\end{aligned}
$$

An important additional feature of this approach is that one separates the scattered and incident wave components. Because of the symmetry of the operations, it is a trivial extension to use this method to introduce arbitrary EM waves into the simulation domain. We have implemented both the $1^{\text {st }}$ and $2^{\text {nd }}$ advection methods in the MAGIC software. In the remaining discussion we will refer to the original matched phase velocity method used in MAGIC as MPV (denoting the original non-centered model), and
CMPV1, (denoting the $1^{\text {st }}$ order advection), and CMPV2, (denoting the $2^{\text {nd }}$ order advection update), and so forth.

\section{THE CYCLOTRON AUTO-RESONANT MASER (CARM) AS A SAMPLE PROBLEM}

As an example problem, we will use the simple geometry of Fig. (2) to represent a cyclotron auto-resonant maser [8]. A CARM is a compact high efficiency, high frequency device. In the maser, electrons gyrating in a circular orbit interact with a rotating electric field. Interaction of the electron beam of a particular gyro-frequency and an RF field of the same (or nearly the same) frequency results in amplification of the RF signal as beam energy is converted to RF energy. Practical efficiencies in energy conversion may be as much as $40 \%$. In our example, we use a hollow cylindrical guide, with an $11 \mathrm{~mm}$ diameter, and a $70-\mathrm{mm}$ length.

In the cold test problem we inject a TE01 excitation signal of $103 \mathrm{GHz}$ at the left side of the test guide.

The Standing Wave Ratio (SWR) of the $E_{\phi}$ field is measured along the interior of the guide at a half radius between $\mathrm{z}=1 \mathrm{~cm}$ and $6 \mathrm{~cm}$. The square of the field is integrated over $\mathrm{T}=1 \mathrm{RF}$ period.

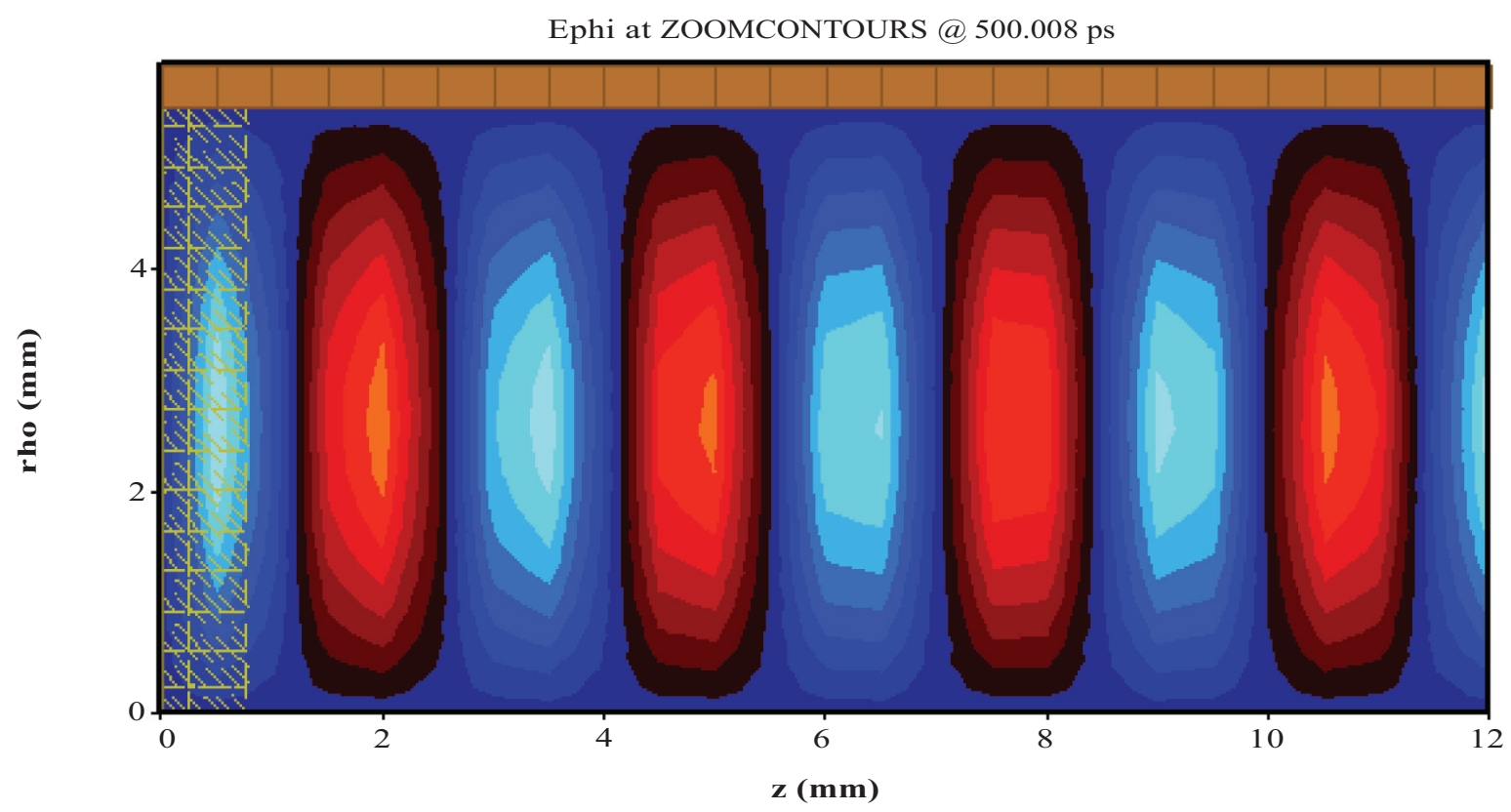

Fig. (3). Illustrates the effect of coarse resolution of the axial mesh on the Te01 field. 


$$
\begin{gathered}
<E_{\phi}(z)>=\left[\left(\frac{2}{T}\right) \int_{0}^{T} E_{\phi}(z, t)^{2} d t\right]^{1 / 2} \\
\mathrm{~S}=\mathrm{SWR}=\operatorname{Max}\left(<E_{\phi}(z)>\right) / \operatorname{Min}\left(<E_{\phi}(z)>\right) \\
|\mathrm{S} 11|=(\mathrm{S}-1) /(\mathrm{S}+1)
\end{gathered}
$$

Measurements were taken for several different grid resolutions. Table $\mathbf{1}$ below lists the resolution parameters. The notation, R4,2 (for example), abbreviates the ratio of zone sizes of one fourth in $\mathrm{z}$ and one half in $\mathrm{r}$ employed compared to the baseline calculation. This ratio is used elsewhere in the paper to characterize numerical resolution, as well.
Table 1. Table of Mesh Resolution Parameters

\begin{tabular}{|c|c|c|c|c|}
\hline Case & Resolution & $\begin{array}{c}\delta \mathbf{r} \\
(\mathbf{m m})\end{array}$ & $\begin{array}{c}\delta \mathbf{z} \\
(\mathbf{m m})\end{array}$ & $\boldsymbol{\lambda} / \boldsymbol{\delta} \mathbf{z}$ \\
\hline \hline 1 & Coarse $($ baseline $)$ & 0.36 & 0.5 & 5.81 \\
\hline 2 & $\mathrm{R} 2\left(\delta \mathrm{z}_{\text {baseline }} / 2, \delta \mathrm{r}_{\text {baseline }} / 1.44\right)$ & 0.25 & 0.25 & 11.62 \\
\hline 3 & $\mathrm{R} 4\left(\delta \mathrm{z}_{\text {baseline }} / 4, \delta \mathrm{r}_{\text {baseline }} / 1.44\right)$ & 0.25 & 0.125 & 23.23 \\
\hline 4 & $\mathrm{R} 4,2\left(\delta \mathrm{z}_{\text {baseline }} / 4, \delta \mathrm{r}_{\text {baseline }} / 2.00\right)$ & 0.18 & 0.125 & 23.23 \\
\hline 5 & $\mathrm{R} 6,3\left(\delta \mathrm{z}_{\text {baseline }} / 6, \delta \mathrm{r}_{\text {baseline }} / 3.60\right)$ & 0.10 & 0.0833 & 34.87 \\
\hline
\end{tabular}

Figs. $(3,4)$ illustrate the effect of the axial and radial resolution on the $\mathrm{E}_{\phi}$ field. The primary effect is the inadequate resolution of the axial wavelength.

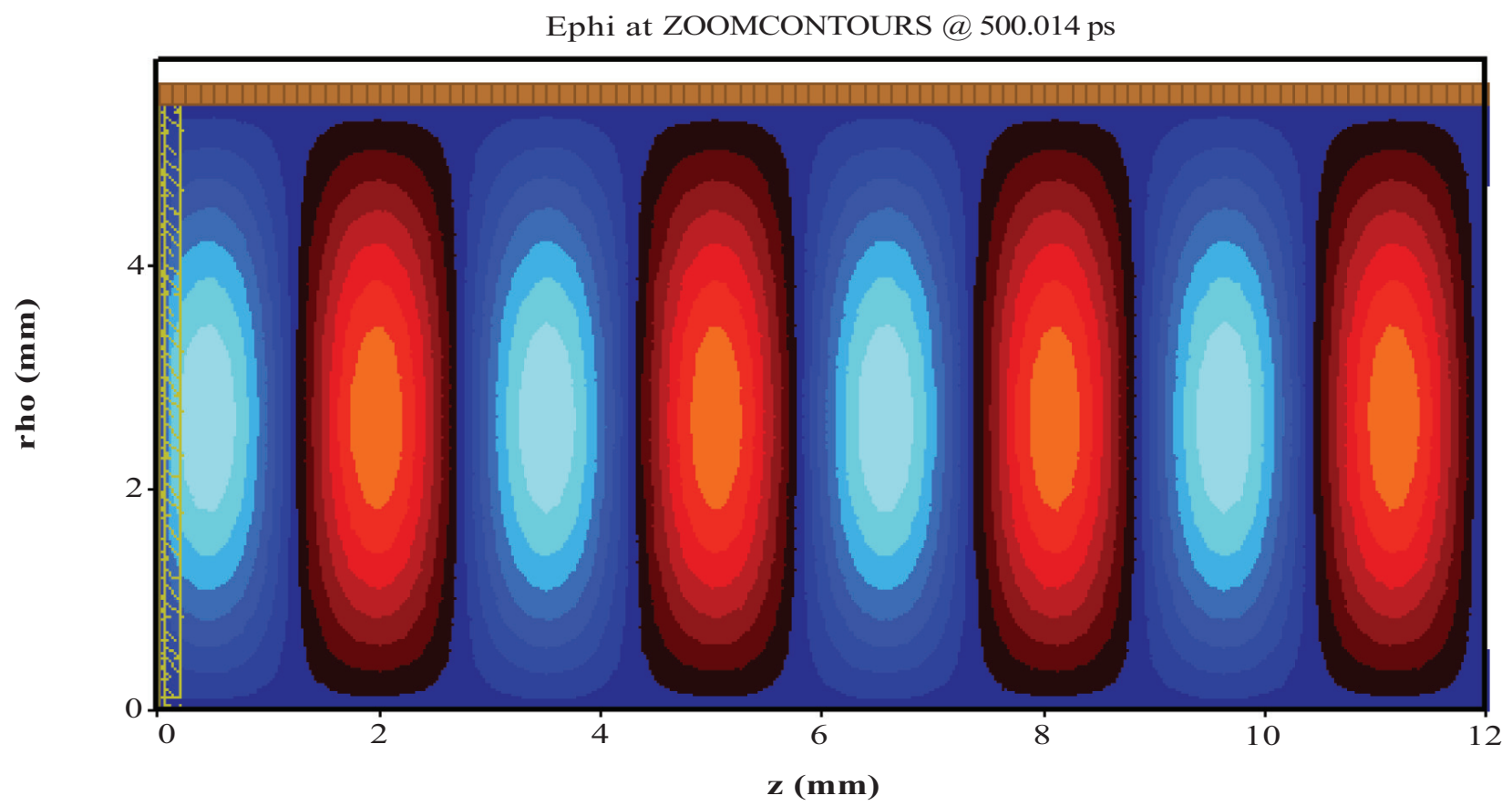

Fig. (4). Illustrates the effect of adequate resolution, Case 4, of the axial and radial mesh on the Te01 field.

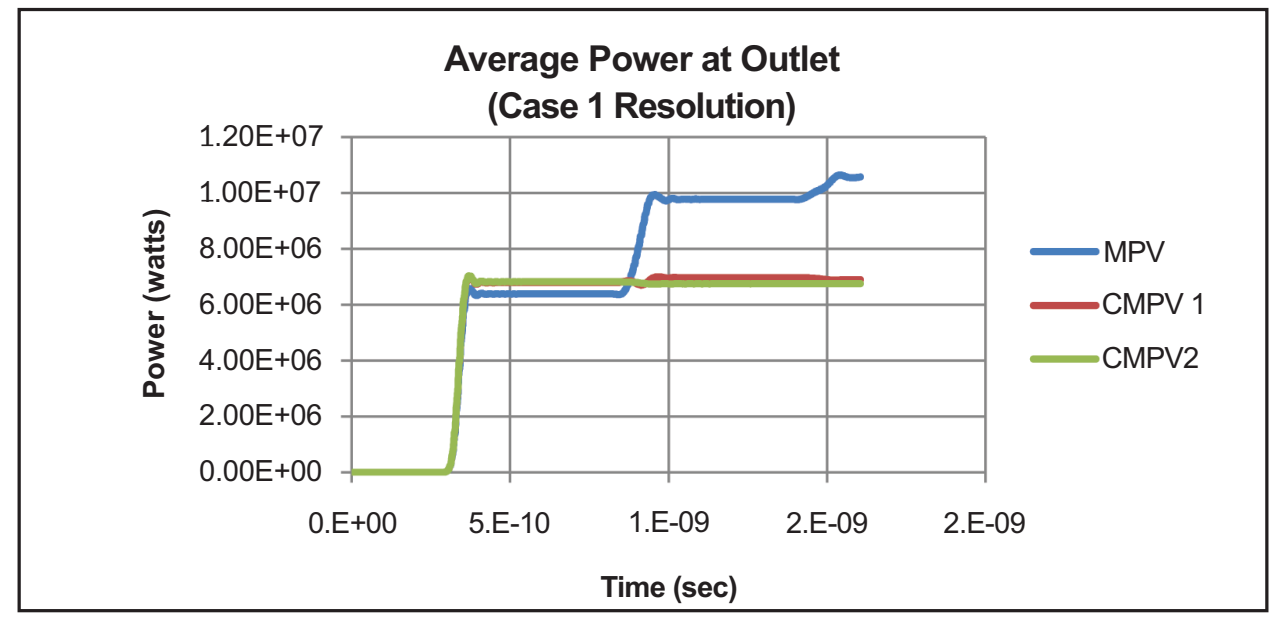

Fig. (5). In this figure we compare radiated average power at the end of the guide for the MPV method, the $1^{\text {st }}$ order CMPV method and the 2nd order CMPV method for Case1 (coarse FD resolution.) 
We determine the reflection from the boundary by measuring the standing wave ratio (SWR) of the electric field amplitude at the half-radius of the cylindrical wave guide along the axial extent of the wave guide. Measurements of the SWR along the interior of the waveguide are given in Table $\mathbf{2}$.

Table 2. S11 from Port Mismatch for Various Grid Resolutions for the 3 Different Matching Models

\begin{tabular}{|c|c|c|c|c|}
\hline Case & Resolution & MPV & $\begin{array}{c}\text { CMPV1 } \\
\mathbf{1}^{\text {st }} \text { Order }\end{array}$ & $\begin{array}{c}\text { CMPV2 } \\
\mathbf{2}^{\text {nd }} \text { Order }\end{array}$ \\
\hline \hline 1 & Coarse & $-11.5 \mathrm{~dB}$ & $-22.4 \mathrm{~dB}$ & $-39.2 \mathrm{~dB}$ \\
\hline 2 & $\mathrm{R} 2$ & $-18.8 \mathrm{~dB}$ & $-33.8 \mathrm{~dB}$ & $-42.4 \mathrm{~dB}$ \\
\hline 3 & $\mathrm{R} 4$ & $-26.1 \mathrm{~dB}$ & $-40.6 \mathrm{~dB}$ & $-43.1 \mathrm{~dB}$ \\
\hline 4 & $\mathrm{R} 4,2$ & $-26.9 \mathrm{~dB}$ & $-42.0 \mathrm{~dB}$ & $-45.9 \mathrm{~dB}$ \\
\hline 5 & $\mathrm{R} 6,3$ & $-28.9 \mathrm{~dB}$ & $-47.7 \mathrm{~dB}$ & $-50.9 \mathrm{db}$ \\
\hline
\end{tabular}

As can be seen in the table the $2^{\text {nd }}$ order CMPV method gives exceptionally good matching regardless of the resolution of the mesh. The $1^{\text {st }}$ order CMPV method matches that of the $2^{\text {nd }}$ order when the axial resolution is improved by a factor of 4 . The older MPV model gives adequate matching at the higher resolutions but never achieves a better match than about $-29 \mathrm{~dB}$, which nevertheless would be satisfactory in many hot test applications.

Fig. (5) shows the average output power for Case 1 and illustrates the effect of poor boundary matching caused by coarse resolution.

Fig. (6) shows the improvement in the behavior of the standard model (MPV) when the resolution of the grid allows a good representation of the wavelength. In this case we note that the both the CMPV1 and CMPV2 appear indistinguishable on this scale.

The value of the improved matching may be demonstrated by injecting a gyro magnetic beam from the left side of the simulation. We are modeling a Cyclotron Auto Resonant Maser (CARM). In this example, we are going to use it as an amplifier of the test signal injected at the right of the simulation. The beam is injected with energy $763 \mathrm{kV}$, current of $2.5 \mathrm{kA}$, and a guiding magnetic field of 2 tesla.

Figs. (7-9) reveal some of the hazards of poor resolution and inadequate matching of the boundary as the reflections from the boundary propagate back upstream of the beam (Fig. 8). Fig. (9) shows the value of adequate resolution with a good boundary match.

Table 3 illustrates the effect of the boundary models on the signal gain. The effects are much as one would expect. Interestingly, for this example problem the damage to the gain in the worst case is not as severe as might be expected from the poor resolution. In fact, the $2^{\text {nd }}$ order advection model is largely insensitive to the marginal resolution case.

Table 3. Table of Signal Gain for the 3 Boundary Models for Different Mesh Resolutions

\begin{tabular}{|c|c|c|c|c|}
\hline Case & Resolution & Gain 0 & Gain 1 & Gain 2 \\
\hline \hline 1 & Coarse & $9.79 \mathrm{~dB}$ & $10.85 \mathrm{~dB}$ & $11.87 \mathrm{~dB}$ \\
\hline 2 & $\mathrm{R} 2$ & $12.55 \mathrm{~dB}$ & $12.55 \mathrm{~dB}$ & $12.70 \mathrm{~dB}$ \\
\hline 4 & $\mathrm{R} 4,2$ & $11.45 \mathrm{~dB}$ & $11.60 \mathrm{~dB}$ & $12.40 \mathrm{~dB}$ \\
\hline
\end{tabular}

\section{CONCLUSIONS}

We began our investigation with the goal of achieving better boundary matching in the cases of both cold test

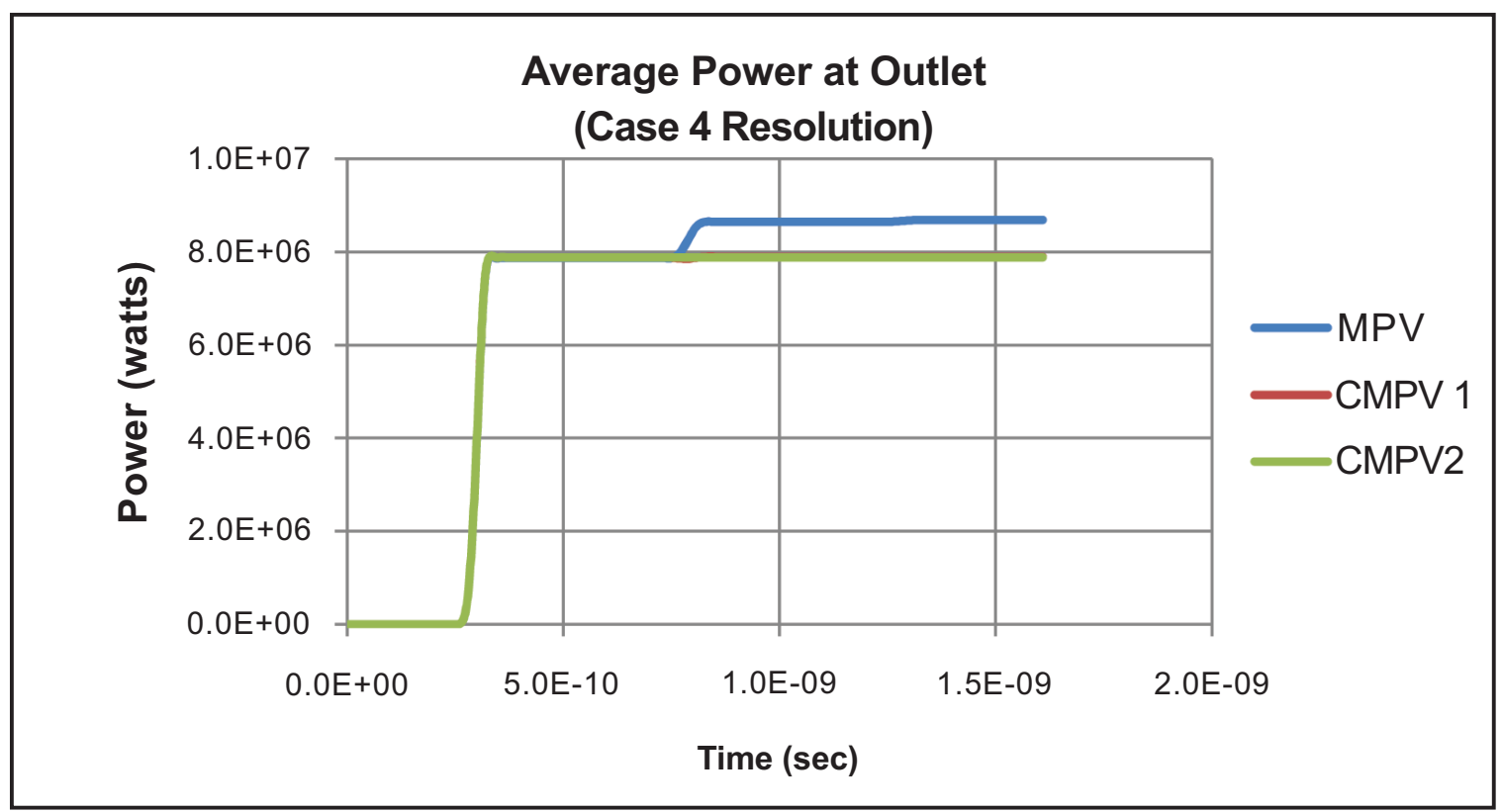

Fig. (6). In this figure we compare radiated average power at the end of the guide for the MPV method, the $1^{\text {st }}$ order CMPV method and the 2nd order CMPV method for Case 4 (Moderate Resolution.) 


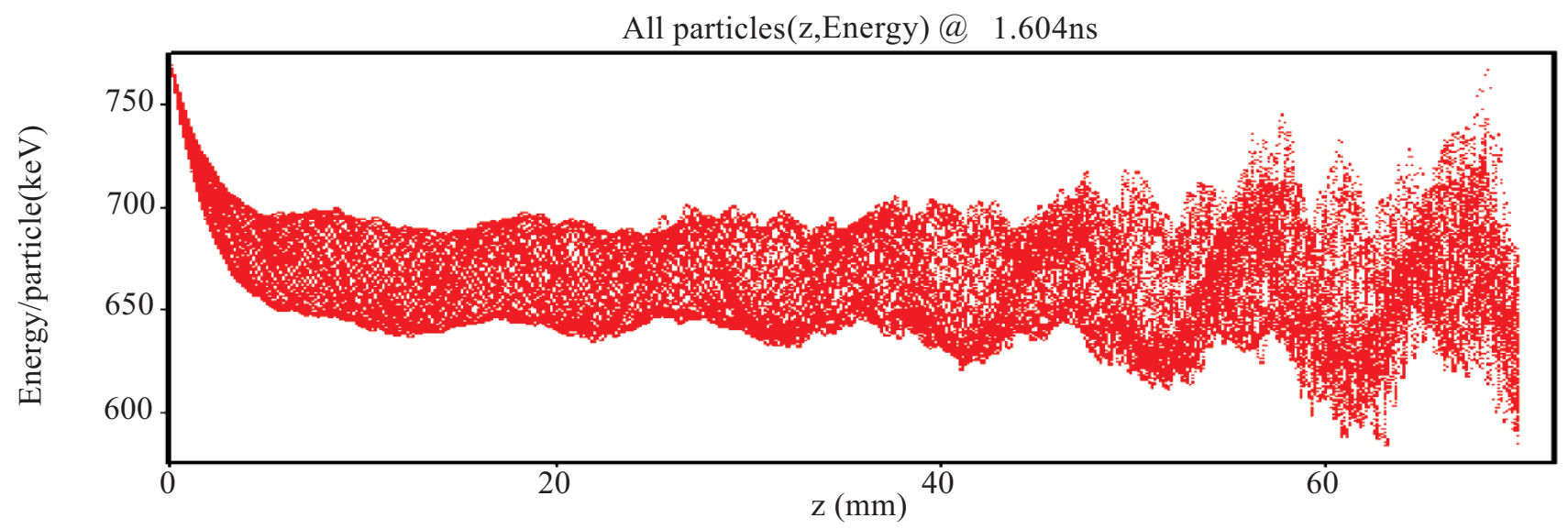

Fig. (7). The base line kinetic energy phase space without an injected signal for coarse resolution illustrates the damage to the beam propagation when using marginal resolution.

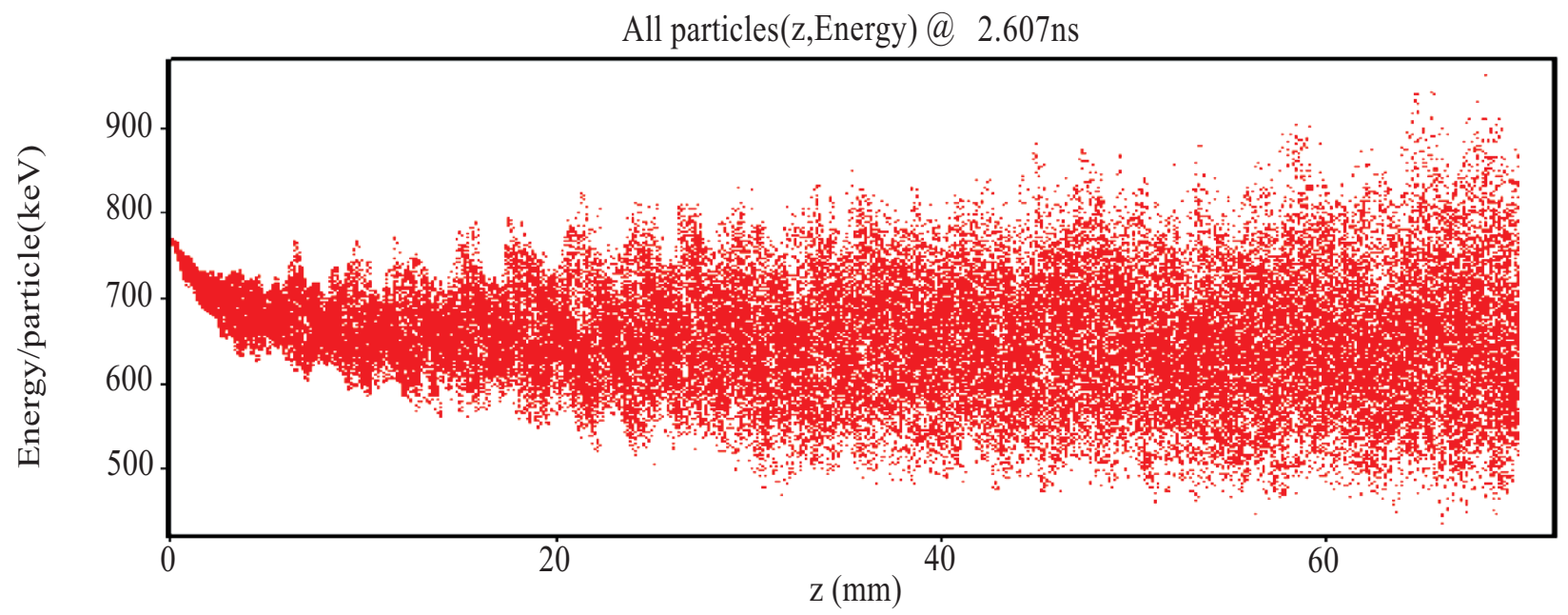

Fig. (8). The kinetic energy phase space with an injected signal for coarse resolution illustrates the damage to the beam propagation when using marginal resolution and the inadequate phase bunching.

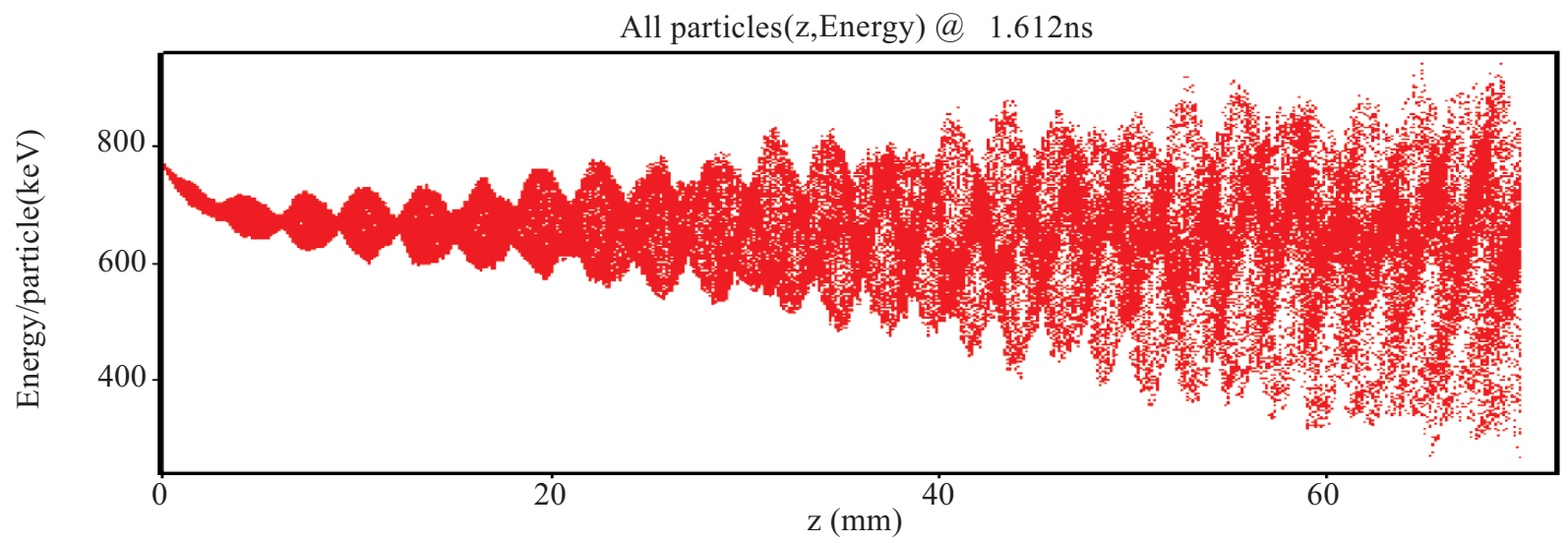

Fig. (9). The kinetic energy phase space with an injected signal for good $(4,2)$ resolution illustrates the amplification and bunching of the beam. The beam is phase bunched with the input signal and transfers energy to the RF. 
performance and in the more extreme case of hot test behaviour. The cold test performance achieves our goal by greatly reducing the artificial reflection from the numerical boundary. In the case of amplifiers this is an important consequence as we wish to reduce feedback effects that can damage performance. For the hot test, in which we permit particles to exit the simulation, we find no ill effects and in fact see that the better performance of the boundary gives somewhat greater gain. The critical finding is that we have not damaged the simulation behaviour by allowing particles to penetrate the boundary.

It is clear that the Centered Matched Phase Velocity Method (CMPV) has greatly improved performance as boundary than does the original Matched Phase Velocity Method (MPV). The effect of $1^{\text {st }}$ order versus $2^{\text {nd }}$ order diminishes as the FD resolution becomes greater and greater. In all cases the CMPV performs better than does the MPV alone.

The $3^{\text {rd }}$ order update was only recently developed and is under testing. The initial results indicate that it provides little improvement over $2^{\text {nd }}$ order when the spatial resolution is reasonably good. The investigation of this approach continues.

\section{ACKNOWLEDGEMENT}

This work expands on research originally sponsored by the Air Force Office of Scientific Research under contract No. FA9550-60-C-0148

\section{REFERENCES}

[1] Analytic Absorbing Boundary Conditions. In: Taflove A, Hagness S, Eds. Computational Electrodynamics, The Finite Difference Time Domain Method, $3^{\text {rd }}$ ed. Artech House 2005; pp. 229-72.

[2] Goplen B, Ludeking L, Smithe D, Warren G. User-configurable MAGIC for electromagnetic PIC calculations. Comput Phys Commun 1995; 87: 54-86 (see also www.mrcwdc.com).

[3] Birdsall CK, Langdon AB. Plasma Physics via Computer Simulation. Taylor and Francis: UK 2005; pp. 369-73.

[4] Schneider J. Advection Equation Approach. Lecture notes EE 417/517. Available at: http://school.eecs.wsu.edu/

[5] Engquist B, Majda A. Absorbing Boundary Conditions of the Numerical Simulation of Waves. Math Comput 1977; 31: 629-51.

[6] Lindman EL. Free-Space boundary conditions for the time dependent wave equation. J Comput Phys 1975; 18: 66-78.

[7] Perfectly Matched Layer Absorbing Boundary Conditions. In: Taflove A, Hagness S, Eds. Computational Electrodynamics, The Finite Difference Time Domain Method, $3^{\text {rd }}$ ed. Artech House 2005; pp. 229-72.

[8] Wang Q, McDermott DB, Luhmann NC. Magnetically tapered CARM (cyclotron autoresonance maser) for high power (Proceedings Paper), in Proceedings, Intense Microwave and Particle Beams II April 1991; vol. 1407: pp. 209-16. 